\title{
Correction to: Improving multi-criterion optimization with chaos: a novel Multi-Objective Chaotic Crow Search Algorithm
}

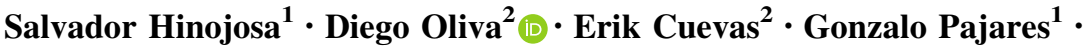 \\ Omar Avalos ${ }^{2} \cdot$ Jorge Gálvez ${ }^{2}$
}

Published online: 2 December 2017

(C) The Natural Computing Applications Forum 2017

\section{Correction to: Neural Comput \& Applic https://doi.org/10.1007/s00521-017-3251-x}

In the original publication, Algorithm 1 and Algorithm 2 are incorrectly published with the same content.

The correct content for Algorithm 1 and Algorithm 2 are given below. The original publication of the article has been updated of the same.

The original article can be found online at https:// doi.org/10.1007/s00521-017-3251-x.

Diego Oliva

diego.oliva@cucei.udg.mx; diego.oliva@itesm.mx

Salvador Hinojosa

salvahin@ucm.es

Erik Cuevas

erik.cuevas@cucei.udg.mx

Gonzalo Pajares

pajares@ucm.es

Omar Avalos

omar.avalos@cucei.udg.mx

Jorge Gálvez

jorge.galvez@cucei.udg.mx

1 Dpto. Ingeniería del Software e Inteligencia Artificial, Facultad Informática, Universidad Complutense de Madrid, 28040 Madrid, Spain

2 División de Electrónica y Computación, Universidad de Guadalajara, CUCEI, Av. Revolución 1500, Guadalajara, Jal, Mexico

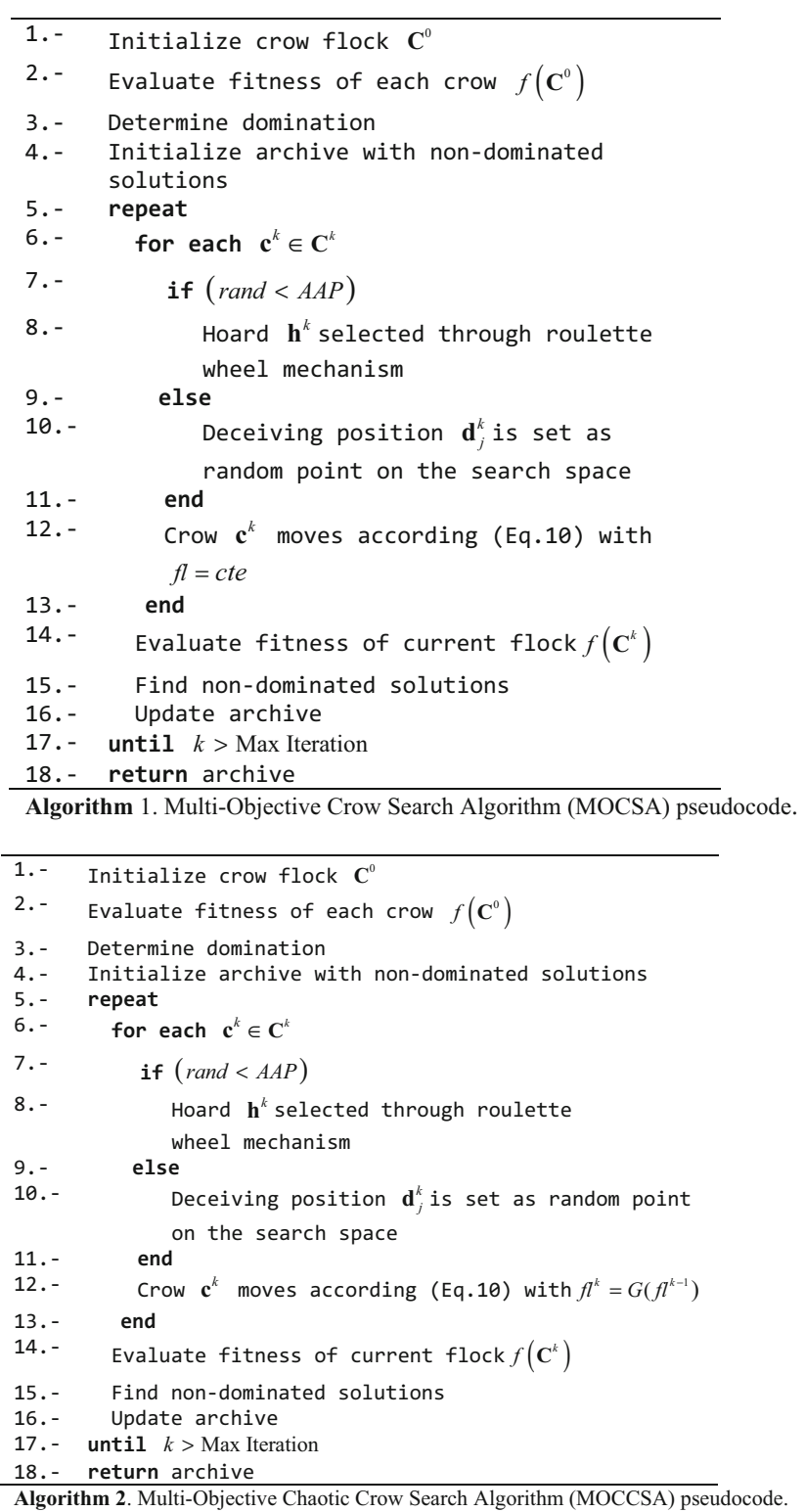

